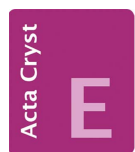

CRYSTALLOGRAPHIC COMMUNICATIONS

ISSN 2056-9890

Received 6 September 2016

Accepted 20 September 2016

Edited by W. T. A. Harrison, University of Aberdeen, Scotland

Keywords: crystal structure; sulfonylurea herbicide; azimsulfuron; dimorphism.

CCDC reference: 1505302

Supporting information: this article has supporting information at journals.iucr.org/e

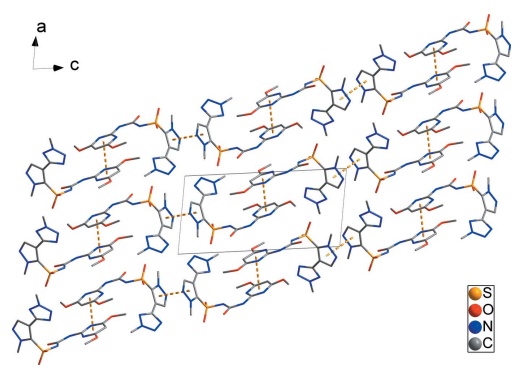

\section{A second triclinic polymorph of azimsulfuron}

\author{
Eunjin Kwon, Jineun Kim, ${ }^{*}$ Hyunjin Park and Tae Ho Kim*
}

Department of Chemistry (BK21 plus) and Research Institute of Natural Sciences, Gyeongsang National University, Jinju 52828, Republic of Korea. *Correspondence e-mail: thkim@gnu.ac.kr, jekim@gnu.ac.kr

The title compound, $\mathrm{C}_{13} \mathrm{H}_{16} \mathrm{~N}_{10} \mathrm{O}_{5} \mathrm{~S}$ (systematic name: 1-(4,6-dimethoxypyrimidin-2-yl)-3-\{[1-methyl-4-(2-methyl-2 $H$-tetrazol-5-yl)pyrazol-5-yl]sulfonyl\}urea), is a second triclinic polymorph of this crystal [for the other, see: Jeon et al., (2015). Acta Cryst. E71, o470-0471]. There are two molecules, $A$ and $B$, in the asymmetric unit; the dihedral angles between the pyrazole ring and the tetrazole and dimethoxypyrimidine ring planes are $72.84(10)$ and $37.24(14)^{\circ}$, respectively (molecule $A$ ) and $84.38(9)$ and $26.09(15)^{\circ}$, respectively (molecule $B)$. Each molecule features an intramolecular $\mathrm{N}-\mathrm{H} \cdots \mathrm{N}$ hydrogen bond. In the crystal, aromatic $\pi-\pi$ stacking interactions [centroid-centroid separations $=$ 3.9871 (16), 3.4487 (14) and 3.5455 (16) §] link the molecules into [001] chains. In addition, $\mathrm{N}-\mathrm{H} \cdots \mathrm{N}, \mathrm{N}-\mathrm{H} \cdots \mathrm{O}, \mathrm{C}-\mathrm{H} \cdots \mathrm{O}$ and $\mathrm{C}-\mathrm{H} \cdots \mathrm{N}$ hydrogen bonds occur, forming a three-dimensional architecture. We propose that the dimorphism results from differences in conformations and packing owing to different intermolecular interactions, especially aromatic $\pi-\pi$ stacking.

\section{Chemical context}

Sulfonylurea herbicides are well known as being highly beneficial for controlling undesirable vegetation in agronomically desirable crops including corn and cereals such as wheat and barley. Azimsulfuron is a recently introduced highly selective sulfonylurea herbicide (Valle et al., 2006) and has been found to be particularly useful as a post-emergent herbicide for weed control in rice paddies and suppression of barnyard grass in rice (Venkatesh et al., 2016). The crystal structure of azimsulfuron (dimorph I) has already been reported in our previous study (Jeon et al., 2015). We now report the crystal structure of a second triclinic polymorph, grown under different conditions, as observed for other systems (Schmidt \& Jansen, 2012; Ebenezer \& Muthiah, 2010).

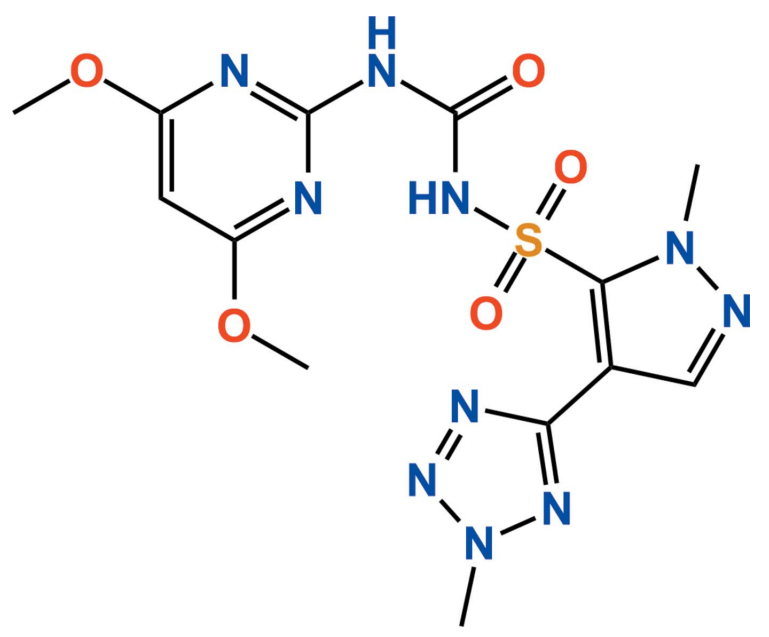




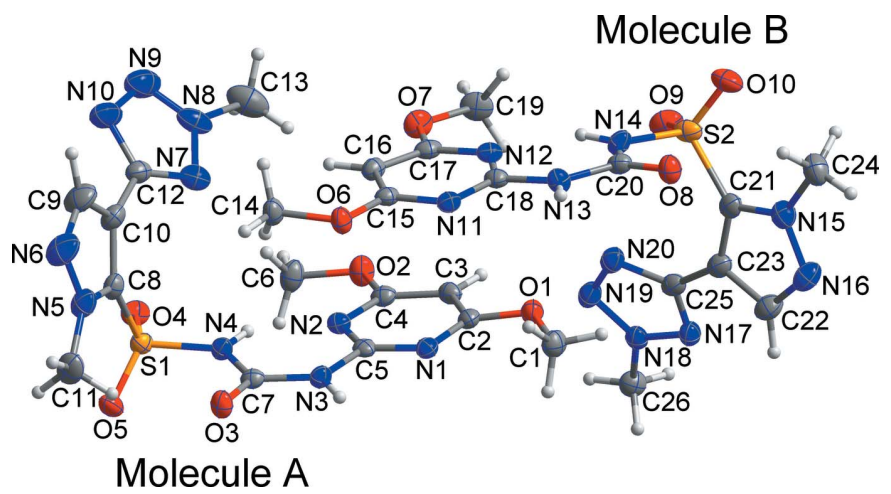

Figure 1

The asymmetric unit of the title compound, with displacement ellipsoids drawn at the $50 \%$ probability level. $\mathrm{H}$ atoms are shown as small spheres of arbitrary radius.
Table 1

Hydrogen-bond geometry $\left(\AA,^{\circ}\right)$.

\begin{tabular}{lllll}
\hline$D-\mathrm{H} \cdots A$ & $D-\mathrm{H}$ & $\mathrm{H} \cdots A$ & $D \cdots A$ & $D-\mathrm{H} \cdots A$ \\
\hline $\mathrm{N} 3-\mathrm{H} 3 N \cdots \mathrm{N} 11^{\mathrm{i}}$ & 0.88 & 2.65 & $3.494(3)$ & 162 \\
$\mathrm{~N} 4-\mathrm{H} 4 N \cdots \mathrm{N} 2$ & 0.88 & 1.89 & $2.611(3)$ & 138 \\
$\mathrm{~N} 13-\mathrm{H} 13 N \cdots \mathrm{O} 3^{\mathrm{i}}$ & 0.88 & 2.04 & $2.844(2)$ & 151 \\
$\mathrm{~N} 14-\mathrm{H} 14 N \cdots \mathrm{N} 12$ & 0.88 & 1.95 & $2.636(3)$ & 134 \\
$\mathrm{C} 1-\mathrm{H} 1 C \cdots \mathrm{O} 8^{\mathrm{ii}}$ & 0.98 & 2.58 & $3.559(3)$ & 178 \\
$\mathrm{C} 11-\mathrm{H} 11 B \cdots \mathrm{O} 3$ & 0.98 & 2.38 & $3.236(3)$ & 146 \\
$\mathrm{C} 11-\mathrm{H} 11 B \cdots \mathrm{O} 8^{\mathrm{i}}$ & 0.98 & 2.57 & $3.278(3)$ & 129 \\
$\mathrm{C} 11-\mathrm{H} 11 C \cdots \mathrm{N} 10^{\mathrm{iii}}$ & 0.98 & 2.58 & $3.290(4)$ & 130 \\
$\mathrm{C} 13-\mathrm{H} 13 A \cdots \mathrm{O} 9^{\text {iv }}$ & 0.98 & 2.40 & $3.374(4)$ & 175 \\
$\mathrm{C} 14-\mathrm{H} 14 A \cdots \mathrm{O} 5^{\mathrm{v}}$ & 0.98 & 2.39 & $3.335(3)$ & 162 \\
$\mathrm{C} 22-\mathrm{H} 22 \cdots \mathrm{N} 17^{\mathrm{vi}}$ & 0.95 & 2.57 & $3.435(3)$ & 152 \\
$\mathrm{C} 24-\mathrm{H} 24 B \cdots \mathrm{O} 8$ & 0.98 & 2.33 & $3.080(3)$ & 133 \\
$\mathrm{C} 26-\mathrm{H} 26 B \cdots \mathrm{N} 16^{\text {vi }}$ & 0.98 & 2.52 & $3.376(3)$ & 146
\end{tabular}

Symmetry codes: (i) $-x+1,-y+1,-z+1$; (ii) $x-1, y, z$; (iii) $-x+1,-y+1,-z$; (iv) $-x+2,-y,-z+1$; (v) $x+1, y, z$; (vi) $-x+1,-y,-z+2$.

\section{Supramolecular features}

In the crystal, aromatic $\pi-\pi$ stacking interactions, $C g 1 \cdots C g 1^{\mathrm{iii}}$ $=3.9817(16), C g 3 \cdots C g 6^{\text {vii }}=3.4487(14)$ and $C g 4 \cdots C g 4^{\text {viii }}=$ 3.5455 (16) $\AA$ occur [Cg1, Cg3, $C g 4$ and $C g 6$ are the centroids of the N5/N6/C8-C10, N1/N2/C2-C5, N15/N16/C21-C23 and N11/N12/C15-C18 rings, respectively; symmetry codes: (vii) $x$, $y, z$, (viii) $-x+2,-y,-z+2$ ]. Together, these link adjacent molecules, forming chains propagating along the $c$ axis. (Fig. 2). In addition, there are $\mathrm{N}-\mathrm{H} \cdots \mathrm{N}, \mathrm{N}-\mathrm{H} \cdots \mathrm{O}, \mathrm{C}-\mathrm{H} \cdots \mathrm{O}$ and $\mathrm{C}-\mathrm{H} \cdots \mathrm{N}$ hydrogen bonds in dimorph II (Table 1), which generate a three-dimensional architecture. hydrogen bond (Table 1), which closes an $S(6)$ ring.

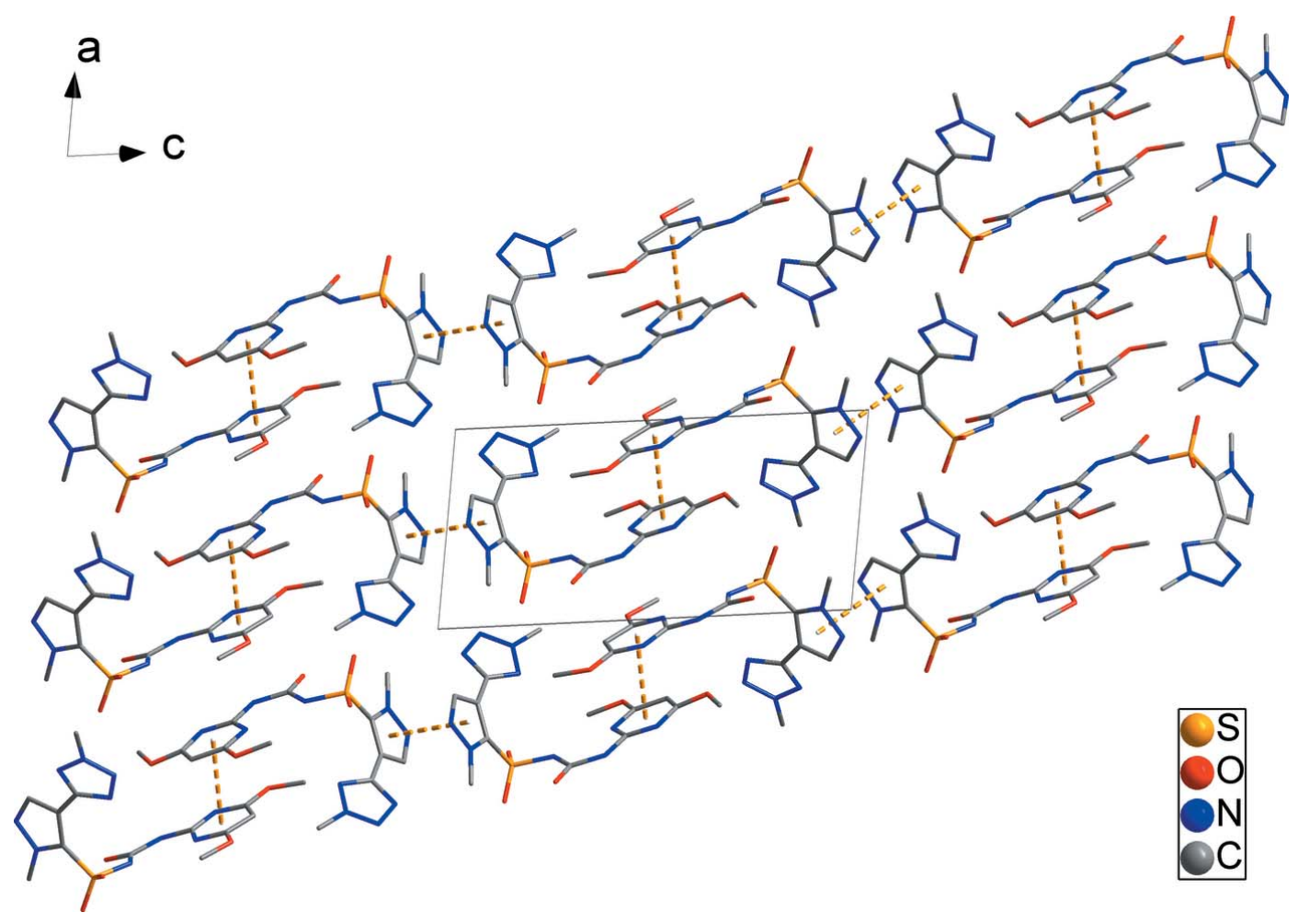

Figure 2

The crystal packing, viewed along the $b$ axis. $\mathrm{H}$ atoms have been omitted for clarity. 
The previous dimorph has only one $\pi-\pi$ interaction between the tetrazole rings of neighboring molecules, while the present dimorph has three $\pi-\pi$ interactions between dimethoxypyrimidine rings in the asymmetric unit, and between the pyrazole rings of neighboring $A$ or $B$ molecules.

\section{Synthesis and crystallization}

In the previous report, crystals were obtained by using $\mathrm{CH}_{3} \mathrm{CN}$ solvent, whereas colourless needles of the title polymorph were prepared by slow evaporation of a methanol solution.

\section{Refinement}

Crystal data, data collection and structure refinement details are summarized in Table 2. All $\mathrm{H}$ atoms were positioned geometrically and refined using a riding model with $d(\mathrm{~N}-\mathrm{H})$ $=0.88 \AA, U_{\text {iso }}=1.2 U_{\text {eq }}(\mathrm{C})$ for $\mathrm{N}-\mathrm{H}$ groups, $d(\mathrm{C}-\mathrm{H})=$ $0.88 \AA, U_{\text {iso }}=1.2 U_{\text {eq }}(\mathrm{C})$ for $\mathrm{Csp} p^{2}-\mathrm{H}$ and $d(\mathrm{C}-\mathrm{H})=0.98 \AA$, $U_{\text {iso }}=1.5 U_{\text {eq }}(\mathrm{C})$ for methyl groups.

\section{Acknowledgements}

This research was supported by the Basic Science Program through the National Research Foundation of Korea (NRF) funded by the Ministry of Education, Science and Technology (No. 2014R1A1A4A01009105).

\section{References}

Brandenburg, K. (2010). DIAMOND. Crystal Impact GbR, Bonn, Germany.

Bruker (2014). APEX2, SAINT and SADABS. Bruker AXS Inc., Madison, Wisconsin, USA.

Chopra, D., Mohan, T. P., Rao, K. S. \& Guru Row, T. N. (2004). Acta Cryst. E60, o2418-o2420.

Ebenezer, S. \& Muthiah, P. T. (2010). Acta Cryst. E66, o2574.

Jeon, Y., Kim, J., Kwon, E. \& Kim, T. H. (2015). Acta Cryst. E71, o470-0471.

Kwon, E., Kim, J., Kang, G. \& Kim, T. H. (2015). Acta Cryst. E71, o1033-o1034.
Table 2

Experimental details.

\begin{tabular}{|c|c|}
\hline \multicolumn{2}{|l|}{ Crystal data } \\
\hline Chemical formula & $\mathrm{C}_{13} \mathrm{H}_{16} \mathrm{~N}_{10} \mathrm{O}_{5} \mathrm{~S}$ \\
\hline$M_{\mathrm{r}}$ & 424.42 \\
\hline Crystal system, space group & Triclinic, $P \overline{1}$ \\
\hline Temperature (K) & 173 \\
\hline$a, b, c(\AA)$ & $7.6451(2), 15.1102(4), 17.0314(5)$ \\
\hline$\alpha, \beta, \gamma\left({ }^{\circ}\right)$ & $\begin{array}{l}67.1562(18), 80.5936(17) \\
84.0996(17)\end{array}$ \\
\hline$V\left(\AA^{3}\right)$ & $1787.08(9)$ \\
\hline$Z$ & 4 \\
\hline Radiation type & Мo $K \alpha$ \\
\hline$\mu\left(\mathrm{mm}^{-1}\right)$ & 0.24 \\
\hline Crystal size $(\mathrm{mm})$ & $0.30 \times 0.04 \times 0.04$ \\
\hline \multicolumn{2}{|l|}{ Data collection } \\
\hline Diffractometer & Bruker APEXII CCD \\
\hline Absorption correction & $\begin{array}{l}\text { Multi-scan (SADABS; Bruker, } \\
\text { 2014) }\end{array}$ \\
\hline$T_{\min }, T_{\max }$ & $0.686,0.746$ \\
\hline $\begin{array}{l}\text { No. of measured, independent and } \\
\text { observed }[I>2 \sigma(I)] \text { reflections }\end{array}$ & $25718,6265,4741$ \\
\hline$R_{\text {int }}$ & 0.060 \\
\hline$(\sin \theta / \lambda)_{\max }\left(\AA^{-1}\right)$ & 0.595 \\
\hline \multicolumn{2}{|l|}{ Refinement } \\
\hline$R\left[F^{2}>2 \sigma\left(F^{2}\right)\right], w R\left(F^{2}\right), S$ & $0.045,0.114,1.07$ \\
\hline No. of reflections & 6265 \\
\hline No. of parameters & 531 \\
\hline $\mathrm{H}$-atom treatment & $\mathrm{H}$-atom parameters constrained \\
\hline$\Delta \rho_{\max }, \Delta \rho_{\min }\left(\mathrm{e} \AA^{-3}\right)$ & $0.38,-0.39$ \\
\hline
\end{tabular}

Computer programs: APEX2 and SAINT (Bruker, 2014), SHELXS97 and SHELXTL (Sheldrick, 2008), SHELXL2014 (Sheldrick, 2015), DIAMOND (Brandenburg, 2010) and publCIF (Westrip, 2010).

Schmidt, C. L. \& Jansen, M. (2012). Z. Anorg. Allg. Chem. 638, 275278.

Sheldrick, G. M. (2008). Acta Cryst. A64, 112-122.

Sheldrick, G. M. (2015). Acta Cryst. C71, 3-8.

Valle, A., Boschin, G., Negri, M., Abbruscato, P., Sorlini, C., D'Agostina, A. \& Zanardini, E. (2006). J. Appl. Microbiol. 101, 443-452.

Venkatesh, P. M., Pardhasaradhi, V., Dhayanithi, V., Avinash, M., Palimkar, S., Subhash, K. R. \& Mohan, H. R. (2016). Chem. Sci. Trans. 5, 41-55.

Westrip, S. P. (2010). J. Appl. Cryst. 43, 920-925. 


\section{supporting information}

Acta Cryst. (2016). E72, 1468-1470 [https://doi.org/10.1107/S2056989016014845]

\section{A second triclinic polymorph of azimsulfuron}

\section{Eunjin Kwon, Jineun Kim, Hyunjin Park and Tae Ho Kim}

Computing details

Data collection: APEX2 (Bruker, 2014); cell refinement: SAINT (Bruker, 2014); data reduction: SAINT (Bruker, 2014); program(s) used to solve structure: SHELXS97 (Sheldrick, 2008); program(s) used to refine structure: SHELXL2014 (Sheldrick, 2015); molecular graphics: DIAMOND (Brandenburg, 2010); software used to prepare material for publication: SHELXTL (Sheldrick, 2008) and publCIF (Westrip, 2010).

1-(4,6-Dimethoxypyrimidin-2-yl)-3-\{[1-methyl-4-(2-methyl-2H-tetrazol-5-yl)pyrazol-5-yl]sulfonyl\}urea

Crystal data

$\mathrm{C}_{13} \mathrm{H}_{16} \mathrm{~N}_{10} \mathrm{O}_{5} \mathrm{~S}$

$M_{r}=424.42$

Triclinic, $P 1$

$a=7.6451(2) \AA$

$b=15.1102(4) \AA$

$c=17.0314(5) \AA$

$\alpha=67.1562(18)^{\circ}$

$\beta=80.5936(17)^{\circ}$

$\gamma=84.0996(17)^{\circ}$

$V=1787.08(9) \AA^{3}$

Data collection

Bruker APEXII CCD

diffractometer

$\varphi$ and $\omega$ scans

Absorption correction: multi-scan

(SADABS; Bruker, 2014)

$T_{\min }=0.686, T_{\max }=0.746$

25718 measured reflections

Refinement

Refinement on $F^{2}$

Least-squares matrix: full

$R\left[F^{2}>2 \sigma\left(F^{2}\right)\right]=0.045$

$w R\left(F^{2}\right)=0.114$

$S=1.07$

6265 reflections

531 parameters

0 restraints
$Z=4$

$F(000)=880$

$D_{\mathrm{x}}=1.577 \mathrm{Mg} \mathrm{m}^{-3}$

Mo $K \alpha$ radiation, $\lambda=0.71073 \AA$

Cell parameters from 3336 reflections

$\theta=2.3-24.2^{\circ}$

$\mu=0.24 \mathrm{~mm}^{-1}$

$T=173 \mathrm{~K}$

Needle, colourless

$0.30 \times 0.04 \times 0.04 \mathrm{~mm}$

6265 independent reflections

4741 reflections with $I>2 \sigma(I)$

$R_{\text {int }}=0.060$

$\theta_{\text {max }}=25.0^{\circ}, \theta_{\text {min }}=1.5^{\circ}$

$h=-8 \rightarrow 9$

$k=-17 \rightarrow 17$

$l=-20 \rightarrow 20$

Hydrogen site location: inferred from neighbouring sites

$\mathrm{H}$-atom parameters constrained

$w=1 /\left[\sigma^{2}\left(F_{\mathrm{o}}^{2}\right)+(0.0457 P)^{2}+0.2151 P\right]$

where $P=\left(F_{\mathrm{o}}{ }^{2}+2 F_{\mathrm{c}}{ }^{2}\right) / 3$

$(\Delta / \sigma)_{\max }<0.001$

$\Delta \rho_{\max }=0.38 \mathrm{e} \AA^{-3}$

$\Delta \rho_{\min }=-0.39$ e $\AA^{-3}$ 


\section{Special details}

Geometry. All esds (except the esd in the dihedral angle between two 1.s. planes) are estimated using the full covariance matrix. The cell esds are taken into account individually in the estimation of esds in distances, angles and torsion angles; correlations between esds in cell parameters are only used when they are defined by crystal symmetry. An approximate (isotropic) treatment of cell esds is used for estimating esds involving l.s. planes.

Fractional atomic coordinates and isotropic or equivalent isotropic displacement parameters $\left(\AA^{2}\right)$

\begin{tabular}{|c|c|c|c|c|}
\hline & $x$ & $y$ & $z$ & $U_{\text {iso }} * / U_{\text {eq }}$ \\
\hline S1 & $0.29659(8)$ & $0.39202(4)$ & $0.20930(4)$ & $0.02210(17)$ \\
\hline S2 & $1.16721(9)$ & $0.10185(4)$ & $0.78289(4)$ & $0.02695(18)$ \\
\hline $\mathrm{O} 1$ & $0.6095(2)$ & $0.23247(11)$ & $0.66818(10)$ & $0.0267(4)$ \\
\hline $\mathrm{O} 2$ & $0.5902(2)$ & 0.10067 (11) & $0.46454(10)$ & $0.0295(4)$ \\
\hline $\mathrm{O} 3$ & $0.2038(2)$ & $0.50940(11)$ & $0.31822(10)$ & $0.0260(4)$ \\
\hline O4 & $0.3602(2)$ & $0.30534(11)$ & $0.19780(11)$ & $0.0288(4)$ \\
\hline O5 & $0.1186(2)$ & $0.42651(12)$ & $0.19829(11)$ & $0.0294(4)$ \\
\hline O6 & $0.7598(2)$ & $0.48627(11)$ & $0.37801(10)$ & $0.0260(4)$ \\
\hline $\mathrm{O} 7$ & $1.0204(2)$ & $0.17015(12)$ & $0.45730(11)$ & $0.0315(4)$ \\
\hline O8 & $1.0811(2)$ & $0.30423(11)$ & $0.75749(11)$ & $0.0285(4)$ \\
\hline O9 & $1.1625(2)$ & $0.01909(11)$ & $0.76253(12)$ & $0.0346(5)$ \\
\hline $\mathrm{O} 10$ & $1.3265(2)$ & $0.12288(12)$ & $0.80359(12)$ & $0.0360(5)$ \\
\hline N1 & $0.4586(3)$ & $0.31656(13)$ & $0.55273(12)$ & $0.0217(5)$ \\
\hline $\mathrm{N} 2$ & $0.4580(3)$ & $0.25049(13)$ & $0.44580(12)$ & $0.0214(5)$ \\
\hline N3 & $0.3252(3)$ & $0.40128(13)$ & $0.43281(12)$ & $0.0210(5)$ \\
\hline $\mathrm{H} 3 \mathrm{~N}$ & 0.2896 & 0.4405 & 0.4598 & $0.025^{*}$ \\
\hline N4 & $0.3313(3)$ & $0.37078(13)$ & $0.30805(12)$ & $0.0212(5)$ \\
\hline $\mathrm{H} 4 \mathrm{~N}$ & 0.3867 & 0.3161 & 0.3356 & $0.025^{*}$ \\
\hline N5 & $0.3879(3)$ & $0.57663(15)$ & $0.10476(13)$ & $0.0327(6)$ \\
\hline N6 & $0.5224(4)$ & $0.62778(16)$ & $0.05092(15)$ & $0.0458(7)$ \\
\hline N7 & $0.7561(3)$ & $0.31962(15)$ & $0.20532(14)$ & $0.0311(5)$ \\
\hline N8 & $0.8972(3)$ & $0.26823(15)$ & $0.18460(16)$ & $0.0357(6)$ \\
\hline N9 & $0.9657(4)$ & $0.3035(2)$ & $0.10352(18)$ & $0.0519(7)$ \\
\hline N10 & $0.8695(3)$ & $0.3815(2)$ & $0.06687(16)$ & $0.0466(7)$ \\
\hline N11 & $0.8673(3)$ & $0.41362(13)$ & $0.50271(12)$ & $0.0219(5)$ \\
\hline N12 & $1.0024(3)$ & $0.25495(13)$ & $0.54475(12)$ & $0.0215(5)$ \\
\hline N13 & $0.9852(3)$ & $0.34673(13)$ & $0.62868(12)$ & $0.0218(5)$ \\
\hline $\mathrm{H} 13 \mathrm{~N}$ & 0.9551 & 0.4035 & 0.6311 & $0.026^{*}$ \\
\hline N14 & $1.1044(3)$ & $0.19100(13)$ & $0.69852(12)$ & $0.0242(5)$ \\
\hline $\mathrm{H} 14 \mathrm{~N}$ & 1.0987 & 0.1805 & 0.6517 & $0.029^{*}$ \\
\hline N15 & $1.0132(3)$ & $0.13158(14)$ & $0.92857(13)$ & $0.0293(5)$ \\
\hline N16 & $0.8644(3)$ & $0.11937(15)$ & $0.98511(14)$ & $0.0350(6)$ \\
\hline N17 & $0.6174(3)$ & $-0.04932(14)$ & $0.89155(12)$ & $0.0247(5)$ \\
\hline N18 & $0.5689(3)$ & $-0.07811(14)$ & $0.83420(13)$ & $0.0241(5)$ \\
\hline N19 & $0.6566(3)$ & $-0.03852(15)$ & $0.75612(13)$ & $0.0298(5)$ \\
\hline $\mathrm{N} 20$ & $0.7690(3)$ & $0.01978(15)$ & $0.76081(13)$ & $0.0295(5)$ \\
\hline $\mathrm{C} 1$ & $0.5501(4)$ & $0.30726(18)$ & $0.70047(16)$ & $0.0298(6)$ \\
\hline $\mathrm{H} 1 \mathrm{~A}$ & 0.5859 & 0.3696 & 0.6566 & $0.045^{*}$ \\
\hline H1B & 0.6036 & 0.2952 & 0.7524 & $0.045^{*}$ \\
\hline
\end{tabular}




\begin{tabular}{|c|c|c|c|c|}
\hline $\mathrm{H} 1 \mathrm{C}$ & 0.4206 & 0.3078 & 0.7143 & $0.045^{*}$ \\
\hline $\mathrm{C} 2$ & $0.5514(3)$ & $0.23781(16)$ & $0.59675(15)$ & $0.0218(6)$ \\
\hline $\mathrm{C} 3$ & $0.5963(3)$ & $0.16081(16)$ & $0.57120(15)$ & $0.0229(6)$ \\
\hline $\mathrm{H} 3$ & 0.6570 & 0.1042 & 0.6051 & $0.028 *$ \\
\hline $\mathrm{C} 4$ & $0.5473(3)$ & $0.17177(16)$ & $0.49379(15)$ & $0.0224(6)$ \\
\hline $\mathrm{C} 5$ & $0.4184(3)$ & $0.31774(16)$ & $0.47966(15)$ & $0.0200(5)$ \\
\hline C6 & $0.5328(4)$ & $0.11182(18)$ & $0.38456(16)$ & $0.0336(7)$ \\
\hline H6A & 0.4032 & 0.1201 & 0.3896 & $0.050^{*}$ \\
\hline H6B & 0.5705 & 0.0545 & 0.3715 & $0.050^{*}$ \\
\hline $\mathrm{H} 6 \mathrm{C}$ & 0.5858 & 0.1684 & 0.3383 & $0.050^{*}$ \\
\hline $\mathrm{C} 7$ & $0.2806(3)$ & $0.43161(16)$ & $0.35078(15)$ & $0.0200(5)$ \\
\hline $\mathrm{C} 8$ & $0.4391(4)$ & $0.48225(17)$ & $0.14203(15)$ & $0.0241(6)$ \\
\hline C9 & 0.6598 (4) & $0.5660(2)$ & $0.05491(18)$ & $0.0427(8)$ \\
\hline H9 & 0.7736 & 0.5832 & 0.0230 & $0.051^{*}$ \\
\hline $\mathrm{C} 10$ & 0.6187 (4) & $0.47261(18)$ & $0.11140(15)$ & $0.0281(6)$ \\
\hline $\mathrm{C} 11$ & 0.2159 (4) & $0.62701(19)$ & $0.11317(18)$ & $0.0393(8)$ \\
\hline H11A & 0.2286 & 0.6964 & 0.0824 & $0.059^{*}$ \\
\hline H11B & 0.1741 & 0.6133 & 0.1741 & $0.059^{*}$ \\
\hline $\mathrm{H} 11 \mathrm{C}$ & 0.1298 & 0.6050 & 0.0887 & $0.059^{*}$ \\
\hline $\mathrm{C} 12$ & $0.7435(3)$ & $0.38992(18)$ & $0.12941(16)$ & $0.0272(6)$ \\
\hline $\mathrm{C} 13$ & 0.9717 (4) & $0.1819(2)$ & $0.2470(2)$ & $0.0522(9)$ \\
\hline H13A & 0.9361 & 0.1250 & 0.2404 & $0.078^{*}$ \\
\hline H13B & 0.9277 & 0.1791 & 0.3053 & $0.078^{*}$ \\
\hline $\mathrm{H} 13 \mathrm{C}$ & 1.1015 & 0.1837 & 0.2374 & $0.078^{*}$ \\
\hline $\mathrm{C} 14$ & $0.7391(4)$ & $0.49082(18)$ & $0.29395(15)$ & $0.0278(6)$ \\
\hline H14A & 0.8562 & 0.4869 & 0.2617 & $0.042 *$ \\
\hline H14B & 0.6774 & 0.5516 & 0.2631 & $0.042 *$ \\
\hline $\mathrm{H} 14 \mathrm{C}$ & 0.6697 & 0.4370 & 0.2998 & $0.042 *$ \\
\hline $\mathrm{C} 15$ & 0.8417 (3) & $0.40702(16)$ & $0.42896(15)$ & $0.0217(6)$ \\
\hline $\mathrm{C} 16$ & $0.8940(3)$ & $0.32688(17)$ & $0.40905(16)$ & $0.0239(6)$ \\
\hline H16 & 0.8781 & 0.3240 & 0.3560 & $0.029 *$ \\
\hline $\mathrm{C} 17$ & $0.9710(3)$ & $0.25142(17)$ & $0.47177(16)$ & $0.0233(6)$ \\
\hline $\mathrm{C} 18$ & $0.9502(3)$ & $0.33713(16)$ & $0.55572(15)$ & $0.0211(6)$ \\
\hline C19 & 1.0937 (4) & $0.09138(17)$ & $0.52594(18)$ & $0.0334(7)$ \\
\hline H19A & 1.1971 & 0.1126 & 0.5401 & $0.050 *$ \\
\hline H19B & 1.1298 & 0.0376 & 0.5074 & $0.050^{*}$ \\
\hline $\mathrm{H} 19 \mathrm{C}$ & 1.0038 & 0.0706 & 0.5768 & $0.050^{*}$ \\
\hline $\mathrm{C} 20$ & $1.0601(3)$ & $0.28177(16)$ & $0.69881(16)$ & $0.0221(6)$ \\
\hline $\mathrm{C} 21$ & $0.9977(3)$ & $0.09825(16)$ & $0.86629(15)$ & $0.0249(6)$ \\
\hline $\mathrm{C} 22$ & $0.7537(4)$ & $0.07751(18)$ & $0.95834(16)$ & $0.0313(7)$ \\
\hline $\mathrm{H} 22$ & 0.6371 & 0.0604 & 0.9865 & $0.038^{*}$ \\
\hline $\mathrm{C} 23$ & $0.8294(4)$ & $0.06179(17)$ & $0.88397(16)$ & $0.0259(6)$ \\
\hline $\mathrm{C} 24$ & $1.1580(4)$ & $0.17922(19)$ & $0.94119(18)$ & $0.0367(7)$ \\
\hline $\mathrm{H} 24 \mathrm{~A}$ & 1.1204 & 0.1980 & 0.9906 & $0.055^{*}$ \\
\hline $\mathrm{H} 24 \mathrm{~B}$ & 1.1880 & 0.2365 & 0.8895 & $0.055^{*}$ \\
\hline $\mathrm{H} 24 \mathrm{C}$ & 1.2623 & 0.1349 & 0.9519 & $0.055^{*}$ \\
\hline $\mathrm{C} 25$ & $0.7428(3)$ & $0.01230(16)$ & $0.84415(15)$ & $0.0246(6)$ \\
\hline $\mathrm{C} 26$ & $0.4366(4)$ & $-0.14953(17)$ & $0.85757(17)$ & $0.0297(6)$ \\
\hline
\end{tabular}




\begin{tabular}{lllll} 
H26A & 0.4199 & -0.1609 & 0.8065 & $0.044^{*}$ \\
H26B & 0.3240 & -0.1263 & 0.8811 & $0.044^{*}$ \\
H26C & 0.4767 & -0.2097 & 0.9009 & $0.044^{*}$ \\
\hline
\end{tabular}

Atomic displacement parameters $\left(\AA^{2}\right)$

\begin{tabular}{|c|c|c|c|c|c|c|}
\hline & $U^{11}$ & $U^{22}$ & $U^{33}$ & $U^{12}$ & $U^{13}$ & $U^{23}$ \\
\hline $\mathrm{S} 1$ & $0.0221(4)$ & 0.0230 & $0.0234(3)$ & $0.0007(3)$ & -0.0029 & $-0.0116(3)$ \\
\hline $\mathrm{S} 2$ & 0.0239 & $0.0192(3)$ & $0.0317(4)$ & $0.0031(3)$ & -0.0010 & -0.0051 \\
\hline O1 & $0.0284(11)$ & $0.0289(9)$ & $0.0222(9)$ & $0.0028(8)$ & $-0.0049(8)$ & $-0.0096(8)$ \\
\hline $\mathrm{O} 2$ & $0.0415(12)$ & $0.0205(9)$ & $0.0287(10)$ & $0.0040(8)$ & $-0.0094(9)$ & $-0.0113(8)$ \\
\hline $\mathrm{O} 3$ & $0.0347(11)$ & $0.0193(9)$ & $0.0233(9)$ & $0.0069(8)$ & $-0.0078(8)$ & $-0.0077(7)$ \\
\hline $\mathrm{O} 4$ & $0.0290(11)$ & $0.0271(9)$ & $0.0368(10)$ & $-0.0005(8)$ & $-0.0028(8)$ & $-0.0199(8)$ \\
\hline O5 & $0.0214(11)$ & $0.0373(10)$ & $0.0323(10)$ & $0.0059(8)$ & $-0.0076(8)$ & $-0.0165(8)$ \\
\hline O6 & $0.0316(11)$ & $0.0263(9)$ & $0.0235(9)$ & $0.0038(8)$ & $-0.0092(8)$ & $-0.0121(7)$ \\
\hline $\mathrm{O} 7$ & $0.0377(12)$ & $0.0265(9)$ & $0.0370(11)$ & $0.0034(8)$ & $-0.0042(9)$ & $-0.0208(8)$ \\
\hline $\mathrm{O} 8$ & $0.0362(12)$ & $0.0238(9)$ & $0.0264(10)$ & $0.0031(8)$ & $-0.0085(8)$ & $-0.0099(8)$ \\
\hline O9 & $0.0368(12)$ & $0.0196(9)$ & $0.0421(11)$ & $0.0040(8)$ & $0.0031(9)$ & $-0.0105(8)$ \\
\hline $\mathrm{O} 10$ & $0.0221(11)$ & $0.0329(10)$ & $0.0472(12)$ & $0.0008(8)$ & $-0.0071(9)$ & $-0.0086(9)$ \\
\hline N1 & $0.0205(12)$ & $0.0218(10)$ & $0.0205(11)$ & $-0.0026(9)$ & $-0.0011(9)$ & $-0.0058(9)$ \\
\hline $\mathrm{N} 2$ & $0.0213(12)$ & $0.0178(10)$ & $0.0232(11)$ & $0.0003(8)$ & $-0.0016(9)$ & $-0.0066(9)$ \\
\hline N3 & $0.0238(12)$ & $0.0198(10)$ & 0.0197 (11) & $0.0026(8)$ & -0.0019 (9) & $-0.0093(8)$ \\
\hline N4 & $0.0246(13)$ & $0.0171(10)$ & $0.0196(10)$ & $0.0053(8)$ & $-0.0032(9)$ & $-0.0057(8)$ \\
\hline N5 & $0.0399(15)$ & $0.0255(11)$ & $0.0251(12)$ & $0.0036(10)$ & $-0.0017(11)$ & $-0.0037(10)$ \\
\hline N6 & 0.0538 (19) & $0.0303(13)$ & $0.0362(14)$ & $-0.0034(12)$ & $0.0064(13)$ & $0.0013(11)$ \\
\hline N7 & $0.0271(14)$ & $0.0277(12)$ & $0.0424(14)$ & $-0.0009(10)$ & $-0.0050(11)$ & $-0.0173(11)$ \\
\hline N8 & $0.0252(14)$ & $0.0316(12)$ & $0.0614(17)$ & $0.0047(10)$ & $-0.0119(12)$ & $-0.0288(12)$ \\
\hline N9 & $0.0380(17)$ & 0.0719 (19) & 0.0605 (19) & 0.0015 (14) & $-0.0009(14)$ & $-0.0443(16)$ \\
\hline N10 & $0.0320(16)$ & $0.0691(18)$ & $0.0476(16)$ & $0.0033(13)$ & $0.0005(12)$ & $-0.0354(14)$ \\
\hline N11 & $0.0210(12)$ & $0.0222(10)$ & $0.0233(11)$ & $-0.0020(9)$ & $-0.0005(9)$ & $-0.0102(9)$ \\
\hline N12 & $0.0188(12)$ & $0.0194(10)$ & 0.0277 (12) & $-0.0023(8)$ & $0.0000(9)$ & $-0.0112(9)$ \\
\hline N13 & $0.0262(13)$ & $0.0164(10)$ & $0.0257(11)$ & $0.0031(8)$ & $-0.0074(9)$ & $-0.0105(9)$ \\
\hline N14 & $0.0302(13)$ & $0.0196(10)$ & $0.0196(11)$ & $0.0033(9)$ & $0.0006(9)$ & $-0.0066(9)$ \\
\hline N15 & $0.0332(15)$ & $0.0229(11)$ & $0.0283(12)$ & $-0.0032(9)$ & $-0.0063(10)$ & $-0.0046(9)$ \\
\hline N16 & $0.0431(16)$ & $0.0301(12)$ & $0.0271(12)$ & $-0.0043(11)$ & $0.0035(11)$ & $-0.0083(10)$ \\
\hline N17 & $0.0276(13)$ & $0.0261(11)$ & $0.0213(11)$ & $0.0023(9)$ & $-0.0047(9)$ & $-0.0103(9)$ \\
\hline N18 & $0.0244(13)$ & $0.0236(11)$ & $0.0244(12)$ & $0.0058(9)$ & $-0.0062(10)$ & $-0.0097(9)$ \\
\hline N19 & $0.0337(15)$ & $0.0309(12)$ & $0.0215(12)$ & $0.0039(10)$ & $-0.0019(10)$ & $-0.0085(10)$ \\
\hline N20 & $0.0323(14)$ & $0.0287(12)$ & $0.0223(12)$ & $0.0013(10)$ & $-0.0022(10)$ & $-0.0055(9)$ \\
\hline $\mathrm{C} 1$ & $0.0329(17)$ & $0.0333(14)$ & 0.0283 (14) & $-0.0024(12)$ & $-0.0032(12)$ & $-0.0171(12)$ \\
\hline $\mathrm{C} 2$ & 0.0183 (14) & $0.0257(13)$ & $0.0189(13)$ & $-0.0025(10)$ & $0.0006(10)$ & $-0.0064(10)$ \\
\hline C3 & $0.0201(15)$ & $0.0198(12)$ & $0.0239(13)$ & $0.0000(10)$ & $-0.0025(11)$ & $-0.0033(10)$ \\
\hline $\mathrm{C} 4$ & $0.0208(15)$ & $0.0191(12)$ & $0.0242(13)$ & $-0.0038(10)$ & 0.0013 (11) & $-0.0057(10)$ \\
\hline $\mathrm{C} 5$ & $0.0153(14)$ & $0.0200(12)$ & $0.0221(13)$ & $-0.0055(10)$ & $0.0001(10)$ & $-0.0049(10)$ \\
\hline C6 & $0.048(2)$ & $0.0288(14)$ & $0.0254(14)$ & $0.0016(13)$ & $-0.0055(13)$ & $-0.0130(12)$ \\
\hline $\mathrm{C} 7$ & $0.0187(14)$ & $0.0172(12)$ & $0.0224(13)$ & $-0.0037(10)$ & $0.0002(10)$ & $-0.0061(10)$ \\
\hline $\mathrm{C} 8$ & $0.0308(16)$ & $0.0236(13)$ & $0.0180(13)$ & $-0.0003(11)$ & $-0.0026(11)$ & $-0.0086(10)$ \\
\hline C9 & $0.045(2)$ & $0.0371(16)$ & $0.0341(17)$ & $-0.0085(14)$ & $0.0087(14)$ & $-0.0040(13)$ \\
\hline
\end{tabular}




\begin{tabular}{lllllll} 
C10 & $0.0319(17)$ & $0.0302(14)$ & $0.0207(13)$ & $-0.0044(12)$ & $0.0026(12)$ & $-0.0098(11)$ \\
C11 & $0.047(2)$ & $0.0314(15)$ & $0.0322(16)$ & $0.0151(13)$ & $-0.0080(14)$ & $-0.0075(13)$ \\
C12 & $0.0226(16)$ & $0.0330(14)$ & $0.0294(15)$ & $-0.0038(11)$ & $0.0002(12)$ & $-0.0164(12)$ \\
C13 & $0.042(2)$ & $0.0348(16)$ & $0.085(3)$ & $0.0064(14)$ & $-0.0173(18)$ & $-0.0268(17)$ \\
C14 & $0.0323(17)$ & $0.0321(14)$ & $0.0224(14)$ & $0.0030(12)$ & $-0.0082(12)$ & $-0.0133(11)$ \\
C15 & $0.0157(14)$ & $0.0254(13)$ & $0.0256(14)$ & $-0.0040(10)$ & $0.0009(11)$ & $-0.0119(11)$ \\
C16 & $0.0229(15)$ & $0.0280(13)$ & $0.0238(13)$ & $-0.0051(11)$ & $0.0001(11)$ & $-0.0133(11)$ \\
C17 & $0.0185(15)$ & $0.0240(13)$ & $0.0304(14)$ & $-0.0047(10)$ & $0.0028(11)$ & $-0.0151(11)$ \\
C18 & $0.0177(14)$ & $0.0198(12)$ & $0.0241(13)$ & $-0.0023(10)$ & $0.0003(11)$ & $-0.0074(10)$ \\
C19 & $0.0363(18)$ & $0.0193(13)$ & $0.0460(17)$ & $0.0026(11)$ & $-0.0045(14)$ & $-0.0150(12)$ \\
C20 & $0.0192(15)$ & $0.0191(12)$ & $0.0249(14)$ & $-0.0002(10)$ & $0.0002(11)$ & $-0.0063(11)$ \\
C21 & $0.0276(16)$ & $0.0201(12)$ & $0.0225(13)$ & $0.0031(11)$ & $-0.0040(11)$ & $-0.0040(10)$ \\
C22 & $0.0332(18)$ & $0.0277(14)$ & $0.0291(15)$ & $-0.0044(12)$ & $0.0031(13)$ & $-0.0085(12)$ \\
C23 & $0.0269(16)$ & $0.0213(12)$ & $0.0249(14)$ & $0.0015(11)$ & $-0.0010(11)$ & $-0.0053(11)$ \\
C24 & $0.0420(19)$ & $0.0344(15)$ & $0.0341(16)$ & $-0.0048(13)$ & $-0.0132(14)$ & $-0.0096(13)$ \\
C25 & $0.0243(16)$ & $0.0198(12)$ & $0.0245(14)$ & $0.0037(11)$ & $-0.0008(11)$ & $-0.0050(11)$ \\
C26 & $0.0312(17)$ & $0.0281(14)$ & $0.0329(15)$ & $-0.0003(12)$ & $-0.0039(12)$ & $-0.0154(12)$ \\
& & & & & & \\
\hline
\end{tabular}

Geometric parameters $\left(\AA,{ }^{\circ}\right)$

\begin{tabular}{llll}
\hline $\mathrm{S} 1-\mathrm{O} 5$ & $1.4212(18)$ & $\mathrm{N} 15-\mathrm{C} 21$ & $1.364(3)$ \\
$\mathrm{S} 1-\mathrm{O} 4$ & $1.4238(16)$ & $\mathrm{N} 15-\mathrm{C} 24$ & $1.467(4)$ \\
$\mathrm{S} 1-\mathrm{N} 4$ & $1.647(2)$ & $\mathrm{N} 16-\mathrm{C} 22$ & $1.336(4)$ \\
$\mathrm{S} 1-\mathrm{C} 8$ & $1.745(3)$ & $\mathrm{N} 17-\mathrm{N} 18$ & $1.327(3)$ \\
$\mathrm{S} 2-\mathrm{O} 10$ & $1.420(2)$ & $\mathrm{N} 17-\mathrm{C} 25$ & $1.336(3)$ \\
$\mathrm{S} 2-\mathrm{O} 9$ & $1.4267(18)$ & $\mathrm{N} 18-\mathrm{N} 19$ & $1.323(3)$ \\
$\mathrm{S} 2-\mathrm{N} 14$ & $1.6431(19)$ & $\mathrm{N} 18-\mathrm{C} 26$ & $1.451(3)$ \\
$\mathrm{S} 2-\mathrm{C} 21$ & $1.747(3)$ & $\mathrm{N} 19-\mathrm{N} 20$ & $1.324(3)$ \\
$\mathrm{O} 1-\mathrm{C} 2$ & $1.333(3)$ & $\mathrm{N} 20-\mathrm{C} 25$ & $1.362(3)$ \\
$\mathrm{O} 1-\mathrm{C} 1$ & $1.443(3)$ & $\mathrm{C} 1-\mathrm{H} 1 \mathrm{~A}$ & 0.9800 \\
$\mathrm{O} 2-\mathrm{C} 4$ & $1.342(3)$ & $\mathrm{C} 1-\mathrm{H} 1 \mathrm{~B}$ & 0.9800 \\
$\mathrm{O} 2-\mathrm{C} 6$ & $1.441(3)$ & $\mathrm{C} 1-\mathrm{H} 1 \mathrm{C}$ & 0.9800 \\
$\mathrm{O} 3-\mathrm{C} 7$ & $1.222(3)$ & $\mathrm{C} 2-\mathrm{C} 3$ & $1.387(3)$ \\
$\mathrm{O} 6-\mathrm{C} 15$ & $1.340(3)$ & $\mathrm{C} 3-\mathrm{C} 4$ & $1.373(3)$ \\
$\mathrm{O} 6-\mathrm{C} 14$ & $1.440(3)$ & $\mathrm{C} 3-\mathrm{H} 3$ & 0.9500 \\
$\mathrm{O} 7-\mathrm{C} 17$ & $1.349(3)$ & $\mathrm{C} 6-\mathrm{H} 6 \mathrm{~A}$ & 0.9800 \\
$\mathrm{O} 7-\mathrm{C} 19$ & $1.447(3)$ & $\mathrm{C} 6-\mathrm{H} 6 \mathrm{~B}$ & 0.9800 \\
$\mathrm{O} 8-\mathrm{C} 20$ & $1.211(3)$ & $\mathrm{C} 6-\mathrm{H} 6 \mathrm{C}$ & 0.9800 \\
$\mathrm{~N} 1-\mathrm{C} 5$ & $1.323(3)$ & $\mathrm{C} 8-\mathrm{C} 10$ & $1.400(4)$ \\
$\mathrm{N} 1-\mathrm{C} 2$ & $1.338(3)$ & $\mathrm{C} 9-\mathrm{C} 10$ & $1.398(4)$ \\
$\mathrm{N} 2-\mathrm{C} 5$ & $1.338(3)$ & $\mathrm{C} 9-\mathrm{H} 9$ & 0.9500 \\
$\mathrm{~N} 2-\mathrm{C} 4$ & $1.345(3)$ & $\mathrm{C} 10-\mathrm{C} 12$ & $1.455(3)$ \\
$\mathrm{N} 3-\mathrm{C} 7$ & $1.380(3)$ & $\mathrm{C} 11-\mathrm{H} 11 \mathrm{~A}$ & 0.9800 \\
$\mathrm{~N} 3-\mathrm{C} 5$ & $1.399(3)$ & $\mathrm{C} 11-\mathrm{H} 11 \mathrm{~B}$ & 0.9800 \\
$\mathrm{~N} 3-\mathrm{H} 3 \mathrm{~N}$ & 0.8800 & $\mathrm{C} 11-\mathrm{H} 11 \mathrm{C}$ & 0.9800 \\
$\mathrm{~N} 4-\mathrm{C} 7$ & $1.370(3)$ & $\mathrm{C} 13-\mathrm{H} 13 \mathrm{~A}$ & 0.9800 \\
$\mathrm{~N} 4-\mathrm{H} 4 \mathrm{~N}$ & 0.8800 & $\mathrm{C} 13-\mathrm{H} 13 \mathrm{~B}$ & 0.9800 \\
$\mathrm{~N} 5-\mathrm{N} 6$ & $1.341(3)$ & $\mathrm{C} 13-\mathrm{H} 13 \mathrm{C}$ &
\end{tabular}




\begin{tabular}{|c|c|c|c|}
\hline $\mathrm{N} 5-\mathrm{C} 8$ & $1.361(3)$ & $\mathrm{C} 14-\mathrm{H} 14 \mathrm{~A}$ & 0.9800 \\
\hline $\mathrm{N} 5-\mathrm{C} 11$ & $1.465(3)$ & C14-H14B & 0.9800 \\
\hline $\mathrm{N} 6-\mathrm{C} 9$ & $1.324(4)$ & $\mathrm{C} 14-\mathrm{H} 14 \mathrm{C}$ & 0.9800 \\
\hline $\mathrm{N} 7-\mathrm{C} 12$ & $1.328(3)$ & $\mathrm{C} 15-\mathrm{C} 16$ & $1.384(3)$ \\
\hline $\mathrm{N} 7-\mathrm{N} 8$ & $1.344(3)$ & $\mathrm{C} 16-\mathrm{C} 17$ & $1.382(3)$ \\
\hline N8-N9 & $1.310(3)$ & $\mathrm{C} 16-\mathrm{H} 16$ & 0.9500 \\
\hline $\mathrm{N} 8-\mathrm{C} 13$ & $1.458(4)$ & C19-H19A & 0.9800 \\
\hline N9-N10 & $1.312(3)$ & C19-H19B & 0.9800 \\
\hline $\mathrm{N} 10-\mathrm{C} 12$ & $1.350(3)$ & $\mathrm{C} 19-\mathrm{H} 19 \mathrm{C}$ & 0.9800 \\
\hline $\mathrm{N} 11-\mathrm{C} 18$ & $1.333(3)$ & $\mathrm{C} 21-\mathrm{C} 23$ & $1.393(4)$ \\
\hline $\mathrm{N} 11-\mathrm{C} 15$ & $1.345(3)$ & $\mathrm{C} 22-\mathrm{C} 23$ & $1.400(3)$ \\
\hline $\mathrm{N} 12-\mathrm{C} 17$ & $1.326(3)$ & $\mathrm{C} 22-\mathrm{H} 22$ & 0.9500 \\
\hline $\mathrm{N} 12-\mathrm{C} 18$ & $1.338(3)$ & $\mathrm{C} 23-\mathrm{C} 25$ & $1.449(4)$ \\
\hline $\mathrm{N} 13-\mathrm{C} 18$ & $1.376(3)$ & $\mathrm{C} 24-\mathrm{H} 24 \mathrm{~A}$ & 0.9800 \\
\hline $\mathrm{N} 13-\mathrm{C} 20$ & $1.384(3)$ & $\mathrm{C} 24-\mathrm{H} 24 \mathrm{~B}$ & 0.9800 \\
\hline N13-H13N & 0.8800 & $\mathrm{C} 24-\mathrm{H} 24 \mathrm{C}$ & 0.9800 \\
\hline $\mathrm{N} 14-\mathrm{C} 20$ & $1.380(3)$ & $\mathrm{C} 26-\mathrm{H} 26 \mathrm{~A}$ & 0.9800 \\
\hline $\mathrm{N} 14-\mathrm{H} 14 \mathrm{~N}$ & 0.8800 & $\mathrm{C} 26-\mathrm{H} 26 \mathrm{~B}$ & 0.9800 \\
\hline N15-N16 & $1.343(3)$ & $\mathrm{C} 26-\mathrm{H} 26 \mathrm{C}$ & 0.9800 \\
\hline $\mathrm{O} 5-\mathrm{S} 1-\mathrm{O} 4$ & $120.08(11)$ & $\mathrm{O} 3-\mathrm{C} 7-\mathrm{N} 3$ & $121.2(2)$ \\
\hline $\mathrm{O} 5-\mathrm{S} 1-\mathrm{N} 4$ & $110.06(10)$ & $\mathrm{N} 4-\mathrm{C} 7-\mathrm{N} 3$ & $116.2(2)$ \\
\hline $\mathrm{O} 4-\mathrm{S} 1-\mathrm{N} 4$ & $103.25(10)$ & $\mathrm{N} 5-\mathrm{C} 8-\mathrm{C} 10$ & $107.4(2)$ \\
\hline $\mathrm{O} 5-\mathrm{S} 1-\mathrm{C} 8$ & $108.81(11)$ & $\mathrm{N} 5-\mathrm{C} 8-\mathrm{S} 1$ & $124.3(2)$ \\
\hline $\mathrm{O} 4-\mathrm{S} 1-\mathrm{C} 8$ & $107.74(11)$ & $\mathrm{C} 10-\mathrm{C} 8-\mathrm{S} 1$ & $128.17(18)$ \\
\hline $\mathrm{N} 4-\mathrm{S} 1-\mathrm{C} 8$ & $105.99(11)$ & $\mathrm{N} 6-\mathrm{C} 9-\mathrm{C} 10$ & $112.4(3)$ \\
\hline $\mathrm{O} 10-\mathrm{S} 2-\mathrm{O} 9$ & $119.77(11)$ & $\mathrm{N} 6-\mathrm{C} 9-\mathrm{H} 9$ & 123.8 \\
\hline $\mathrm{O} 10-\mathrm{S} 2-\mathrm{N} 14$ & $110.51(11)$ & $\mathrm{C} 10-\mathrm{C} 9-\mathrm{H} 9$ & 123.8 \\
\hline $\mathrm{O} 9-\mathrm{S} 2-\mathrm{N} 14$ & $103.83(11)$ & $\mathrm{C} 9-\mathrm{C} 10-\mathrm{C} 8$ & $103.2(2)$ \\
\hline $\mathrm{O} 10-\mathrm{S} 2-\mathrm{C} 21$ & $108.05(13)$ & $\mathrm{C} 9-\mathrm{C} 10-\mathrm{C} 12$ & $124.8(3)$ \\
\hline $\mathrm{O} 9-\mathrm{S} 2-\mathrm{C} 21$ & $109.42(12)$ & $\mathrm{C} 8-\mathrm{C} 10-\mathrm{C} 12$ & $132.0(2)$ \\
\hline $\mathrm{N} 14-\mathrm{S} 2-\mathrm{C} 21$ & $104.16(11)$ & $\mathrm{N} 5-\mathrm{C} 11-\mathrm{H} 11 \mathrm{~A}$ & 109.5 \\
\hline $\mathrm{C} 2-\mathrm{O} 1-\mathrm{C} 1$ & $117.82(19)$ & N5-C11-H11B & 109.5 \\
\hline $\mathrm{C} 4-\mathrm{O} 2-\mathrm{C} 6$ & 117.94 (19) & $\mathrm{H} 11 \mathrm{~A}-\mathrm{C} 11-\mathrm{H} 11 \mathrm{~B}$ & 109.5 \\
\hline $\mathrm{C} 15-\mathrm{O} 6-\mathrm{C} 14$ & $117.12(18)$ & $\mathrm{N} 5-\mathrm{C} 11-\mathrm{H} 11 \mathrm{C}$ & 109.5 \\
\hline $\mathrm{C} 17-\mathrm{O} 7-\mathrm{C} 19$ & $116.6(2)$ & $\mathrm{H} 11 \mathrm{~A}-\mathrm{C} 11-\mathrm{H} 11 \mathrm{C}$ & 109.5 \\
\hline $\mathrm{C} 5-\mathrm{N} 1-\mathrm{C} 2$ & $114.6(2)$ & $\mathrm{H} 11 \mathrm{~B}-\mathrm{C} 11-\mathrm{H} 11 \mathrm{C}$ & 109.5 \\
\hline $\mathrm{C} 5-\mathrm{N} 2-\mathrm{C} 4$ & $114.8(2)$ & $\mathrm{N} 7-\mathrm{C} 12-\mathrm{N} 10$ & $112.7(2)$ \\
\hline $\mathrm{C} 7-\mathrm{N} 3-\mathrm{C} 5$ & $129.9(2)$ & $\mathrm{N} 7-\mathrm{C} 12-\mathrm{C} 10$ & $126.6(2)$ \\
\hline $\mathrm{C} 7-\mathrm{N} 3-\mathrm{H} 3 \mathrm{~N}$ & 115.0 & $\mathrm{~N} 10-\mathrm{C} 12-\mathrm{C} 10$ & $120.5(2)$ \\
\hline $\mathrm{C} 5-\mathrm{N} 3-\mathrm{H} 3 \mathrm{~N}$ & 115.0 & $\mathrm{~N} 8-\mathrm{C} 13-\mathrm{H} 13 \mathrm{~A}$ & 109.5 \\
\hline $\mathrm{C} 7-\mathrm{N} 4-\mathrm{S} 1$ & $125.14(16)$ & $\mathrm{N} 8-\mathrm{C} 13-\mathrm{H} 13 \mathrm{~B}$ & 109.5 \\
\hline $\mathrm{C} 7-\mathrm{N} 4-\mathrm{H} 4 \mathrm{~N}$ & 117.4 & $\mathrm{H} 13 \mathrm{~A}-\mathrm{C} 13-\mathrm{H} 13 \mathrm{~B}$ & 109.5 \\
\hline $\mathrm{S} 1-\mathrm{N} 4-\mathrm{H} 4 \mathrm{~N}$ & 117.4 & $\mathrm{~N} 8-\mathrm{C} 13-\mathrm{H} 13 \mathrm{C}$ & 109.5 \\
\hline $\mathrm{N} 6-\mathrm{N} 5-\mathrm{C} 8$ & $111.0(2)$ & $\mathrm{H} 13 \mathrm{~A}-\mathrm{C} 13-\mathrm{H} 13 \mathrm{C}$ & 109.5 \\
\hline $\mathrm{N} 6-\mathrm{N} 5-\mathrm{C} 11$ & $118.1(2)$ & $\mathrm{H} 13 \mathrm{~B}-\mathrm{C} 13-\mathrm{H} 13 \mathrm{C}$ & 109.5 \\
\hline $\mathrm{C} 8-\mathrm{N} 5-\mathrm{C} 11$ & $130.9(2)$ & $\mathrm{O} 6-\mathrm{C} 14-\mathrm{H} 14 \mathrm{~A}$ & 109.5 \\
\hline $\mathrm{C} 9-\mathrm{N} 6-\mathrm{N} 5$ & $105.9(2)$ & $\mathrm{O} 6-\mathrm{C} 14-\mathrm{H} 14 \mathrm{~B}$ & 109.5 \\
\hline
\end{tabular}




\begin{tabular}{|c|c|c|c|}
\hline $\mathrm{C} 12-\mathrm{N} 7-\mathrm{N} 8$ & $100.5(2)$ & $\mathrm{H} 14 \mathrm{~A}-\mathrm{C} 14-\mathrm{H} 14 \mathrm{~B}$ & 109.5 \\
\hline $\mathrm{N} 9-\mathrm{N} 8-\mathrm{N} 7$ & $114.3(2)$ & $\mathrm{O} 6-\mathrm{C} 14-\mathrm{H} 14 \mathrm{C}$ & 109.5 \\
\hline $\mathrm{N} 9-\mathrm{N} 8-\mathrm{C} 13$ & $122.4(2)$ & $\mathrm{H} 14 \mathrm{~A}-\mathrm{C} 14-\mathrm{H} 14 \mathrm{C}$ & 109.5 \\
\hline $\mathrm{N} 7-\mathrm{N} 8-\mathrm{C} 13$ & $123.2(2)$ & $\mathrm{H} 14 \mathrm{~B}-\mathrm{C} 14-\mathrm{H} 14 \mathrm{C}$ & 109.5 \\
\hline N8-N9-N10 & $106.2(2)$ & $\mathrm{O} 6-\mathrm{C} 15-\mathrm{N} 11$ & $112.2(2)$ \\
\hline $\mathrm{N} 9-\mathrm{N} 10-\mathrm{C} 12$ & $106.2(2)$ & $\mathrm{O} 6-\mathrm{C} 15-\mathrm{C} 16$ & $124.5(2)$ \\
\hline $\mathrm{C} 18-\mathrm{N} 11-\mathrm{C} 15$ & $115.0(2)$ & $\mathrm{N} 11-\mathrm{C} 15-\mathrm{C} 16$ & $123.3(2)$ \\
\hline $\mathrm{C} 17-\mathrm{N} 12-\mathrm{C} 18$ & $115.4(2)$ & $\mathrm{C} 17-\mathrm{C} 16-\mathrm{C} 15$ & $115.3(2)$ \\
\hline $\mathrm{C} 18-\mathrm{N} 13-\mathrm{C} 20$ & $130.6(2)$ & $\mathrm{C} 17-\mathrm{C} 16-\mathrm{H} 16$ & 122.3 \\
\hline $\mathrm{C} 18-\mathrm{N} 13-\mathrm{H} 13 \mathrm{~N}$ & 114.7 & $\mathrm{C} 15-\mathrm{C} 16-\mathrm{H} 16$ & 122.3 \\
\hline $\mathrm{C} 20-\mathrm{N} 13-\mathrm{H} 13 \mathrm{~N}$ & 114.7 & $\mathrm{~N} 12-\mathrm{C} 17-\mathrm{O} 7$ & $118.0(2)$ \\
\hline $\mathrm{C} 20-\mathrm{N} 14-\mathrm{S} 2$ & $122.10(18)$ & $\mathrm{N} 12-\mathrm{C} 17-\mathrm{C} 16$ & $123.7(2)$ \\
\hline $\mathrm{C} 20-\mathrm{N} 14-\mathrm{H} 14 \mathrm{~N}$ & 119.0 & $\mathrm{O} 7-\mathrm{C} 17-\mathrm{C} 16$ & $118.3(2)$ \\
\hline $\mathrm{S} 2-\mathrm{N} 14-\mathrm{H} 14 \mathrm{~N}$ & 119.0 & $\mathrm{~N} 11-\mathrm{C} 18-\mathrm{N} 12$ & $127.2(2)$ \\
\hline N16-N15-C21 & $111.3(2)$ & $\mathrm{N} 11-\mathrm{C} 18-\mathrm{N} 13$ & $114.6(2)$ \\
\hline N16-N15-C24 & $117.3(2)$ & $\mathrm{N} 12-\mathrm{C} 18-\mathrm{N} 13$ & $118.2(2)$ \\
\hline $\mathrm{C} 21-\mathrm{N} 15-\mathrm{C} 24$ & $131.4(2)$ & $\mathrm{O} 7-\mathrm{C} 19-\mathrm{H} 19 \mathrm{~A}$ & 109.5 \\
\hline $\mathrm{C} 22-\mathrm{N} 16-\mathrm{N} 15$ & $105.5(2)$ & $\mathrm{O} 7-\mathrm{C} 19-\mathrm{H} 19 \mathrm{~B}$ & 109.5 \\
\hline N18-N17-C25 & $102.1(2)$ & $\mathrm{H} 19 \mathrm{~A}-\mathrm{C} 19-\mathrm{H} 19 \mathrm{~B}$ & 109.5 \\
\hline N19-N18-N17 & $113.8(2)$ & $\mathrm{O} 7-\mathrm{C} 19-\mathrm{H} 19 \mathrm{C}$ & 109.5 \\
\hline N19-N18-C26 & $124.1(2)$ & $\mathrm{H} 19 \mathrm{~A}-\mathrm{C} 19-\mathrm{H} 19 \mathrm{C}$ & 109.5 \\
\hline N17-N18-C26 & $122.0(2)$ & $\mathrm{H} 19 \mathrm{~B}-\mathrm{C} 19-\mathrm{H} 19 \mathrm{C}$ & 109.5 \\
\hline N18-N19-N20 & $106.5(2)$ & $\mathrm{O} 8-\mathrm{C} 20-\mathrm{N} 14$ & $122.8(2)$ \\
\hline N19-N20-C25 & $105.9(2)$ & $\mathrm{O} 8-\mathrm{C} 20-\mathrm{N} 13$ & $120.8(2)$ \\
\hline $\mathrm{O} 1-\mathrm{C} 1-\mathrm{H} 1 \mathrm{~A}$ & 109.5 & $\mathrm{~N} 14-\mathrm{C} 20-\mathrm{N} 13$ & $116.4(2)$ \\
\hline $\mathrm{O} 1-\mathrm{C} 1-\mathrm{H} 1 \mathrm{~B}$ & 109.5 & $\mathrm{~N} 15-\mathrm{C} 21-\mathrm{C} 23$ & $107.4(2)$ \\
\hline $\mathrm{H} 1 \mathrm{~A}-\mathrm{C} 1-\mathrm{H} 1 \mathrm{~B}$ & 109.5 & $\mathrm{~N} 15-\mathrm{C} 21-\mathrm{S} 2$ & $123.5(2)$ \\
\hline $\mathrm{O} 1-\mathrm{C} 1-\mathrm{H} 1 \mathrm{C}$ & 109.5 & $\mathrm{C} 23-\mathrm{C} 21-\mathrm{S} 2$ & $129.0(2)$ \\
\hline $\mathrm{H} 1 \mathrm{~A}-\mathrm{C} 1-\mathrm{H} 1 \mathrm{C}$ & 109.5 & $\mathrm{~N} 16-\mathrm{C} 22-\mathrm{C} 23$ & $112.2(3)$ \\
\hline $\mathrm{H} 1 \mathrm{~B}-\mathrm{C} 1-\mathrm{H} 1 \mathrm{C}$ & 109.5 & $\mathrm{~N} 16-\mathrm{C} 22-\mathrm{H} 22$ & 123.9 \\
\hline $\mathrm{O} 1-\mathrm{C} 2-\mathrm{N} 1$ & $119.3(2)$ & $\mathrm{C} 23-\mathrm{C} 22-\mathrm{H} 22$ & 123.9 \\
\hline $\mathrm{O} 1-\mathrm{C} 2-\mathrm{C} 3$ & $117.0(2)$ & $\mathrm{C} 21-\mathrm{C} 23-\mathrm{C} 22$ & $103.6(2)$ \\
\hline $\mathrm{N} 1-\mathrm{C} 2-\mathrm{C} 3$ & $123.7(2)$ & $\mathrm{C} 21-\mathrm{C} 23-\mathrm{C} 25$ & $132.5(2)$ \\
\hline $\mathrm{C} 4-\mathrm{C} 3-\mathrm{C} 2$ & $115.6(2)$ & $\mathrm{C} 22-\mathrm{C} 23-\mathrm{C} 25$ & $123.8(2)$ \\
\hline $\mathrm{C} 4-\mathrm{C} 3-\mathrm{H} 3$ & 122.2 & $\mathrm{~N} 15-\mathrm{C} 24-\mathrm{H} 24 \mathrm{~A}$ & 109.5 \\
\hline $\mathrm{C} 2-\mathrm{C} 3-\mathrm{H} 3$ & 122.2 & $\mathrm{~N} 15-\mathrm{C} 24-\mathrm{H} 24 \mathrm{~B}$ & 109.5 \\
\hline $\mathrm{O} 2-\mathrm{C} 4-\mathrm{N} 2$ & $118.3(2)$ & $\mathrm{H} 24 \mathrm{~A}-\mathrm{C} 24-\mathrm{H} 24 \mathrm{~B}$ & 109.5 \\
\hline $\mathrm{O} 2-\mathrm{C} 4-\mathrm{C} 3$ & $118.6(2)$ & $\mathrm{N} 15-\mathrm{C} 24-\mathrm{H} 24 \mathrm{C}$ & 109.5 \\
\hline $\mathrm{N} 2-\mathrm{C} 4-\mathrm{C} 3$ & $123.1(2)$ & $\mathrm{H} 24 \mathrm{~A}-\mathrm{C} 24-\mathrm{H} 24 \mathrm{C}$ & 109.5 \\
\hline $\mathrm{N} 1-\mathrm{C} 5-\mathrm{N} 2$ & $128.1(2)$ & $\mathrm{H} 24 \mathrm{~B}-\mathrm{C} 24-\mathrm{H} 24 \mathrm{C}$ & 109.5 \\
\hline $\mathrm{N} 1-\mathrm{C} 5-\mathrm{N} 3$ & $114.3(2)$ & $\mathrm{N} 17-\mathrm{C} 25-\mathrm{N} 20$ & $111.8(2)$ \\
\hline $\mathrm{N} 2-\mathrm{C} 5-\mathrm{N} 3$ & $117.5(2)$ & $\mathrm{N} 17-\mathrm{C} 25-\mathrm{C} 23$ & $119.4(2)$ \\
\hline $\mathrm{O} 2-\mathrm{C} 6-\mathrm{H} 6 \mathrm{~A}$ & 109.5 & $\mathrm{~N} 20-\mathrm{C} 25-\mathrm{C} 23$ & $128.8(2)$ \\
\hline $\mathrm{O} 2-\mathrm{C} 6-\mathrm{H} 6 \mathrm{~B}$ & 109.5 & $\mathrm{~N} 18-\mathrm{C} 26-\mathrm{H} 26 \mathrm{~A}$ & 109.5 \\
\hline $\mathrm{H} 6 \mathrm{~A}-\mathrm{C} 6-\mathrm{H} 6 \mathrm{~B}$ & 109.5 & $\mathrm{~N} 18-\mathrm{C} 26-\mathrm{H} 26 \mathrm{~B}$ & 109.5 \\
\hline $\mathrm{O} 2-\mathrm{C} 6-\mathrm{H} 6 \mathrm{C}$ & 109.5 & $\mathrm{H} 26 \mathrm{~A}-\mathrm{C} 26-\mathrm{H} 26 \mathrm{~B}$ & 109.5 \\
\hline $\mathrm{H} 6 \mathrm{~A}-\mathrm{C} 6-\mathrm{H} 6 \mathrm{C}$ & 109.5 & $\mathrm{~N} 18-\mathrm{C} 26-\mathrm{H} 26 \mathrm{C}$ & 109.5 \\
\hline $\mathrm{H} 6 \mathrm{~B}-\mathrm{C} 6-\mathrm{H} 6 \mathrm{C}$ & 109.5 & $\mathrm{H} 26 \mathrm{~A}-\mathrm{C} 26-\mathrm{H} 26 \mathrm{C}$ & 109.5 \\
\hline
\end{tabular}




\begin{tabular}{|c|c|c|c|}
\hline $\mathrm{O} 3-\mathrm{C} 7-\mathrm{N} 4$ & $122.6(2)$ & $\mathrm{H} 26 \mathrm{~B}-\mathrm{C} 26-\mathrm{H} 26 \mathrm{C}$ & 109.5 \\
\hline $\mathrm{O} 5-\mathrm{S} 1-\mathrm{N} 4-\mathrm{C} 7$ & $-46.2(2)$ & $\mathrm{N} 5-\mathrm{C} 8-\mathrm{C} 10-\mathrm{C} 12$ & $179.0(3)$ \\
\hline $\mathrm{O} 4-\mathrm{S} 1-\mathrm{N} 4-\mathrm{C} 7$ & $-175.6(2)$ & $\mathrm{S} 1-\mathrm{C} 8-\mathrm{C} 10-\mathrm{C} 12$ & $-5.2(5)$ \\
\hline $\mathrm{C} 8-\mathrm{S} 1-\mathrm{N} 4-\mathrm{C} 7$ & $71.3(2)$ & $\mathrm{N} 8-\mathrm{N} 7-\mathrm{C} 12-\mathrm{N} 10$ & $-0.2(3)$ \\
\hline $\mathrm{C} 8-\mathrm{N} 5-\mathrm{N} 6-\mathrm{C} 9$ & $-0.8(3)$ & $\mathrm{N} 8-\mathrm{N} 7-\mathrm{C} 12-\mathrm{C} 10$ & $-176.4(3)$ \\
\hline $\mathrm{C} 11-\mathrm{N} 5-\mathrm{N} 6-\mathrm{C} 9$ & $-179.6(3)$ & $\mathrm{N} 9-\mathrm{N} 10-\mathrm{C} 12-\mathrm{N} 7$ & $0.2(3)$ \\
\hline $\mathrm{C} 12-\mathrm{N} 7-\mathrm{N} 8-\mathrm{N} 9$ & $0.2(3)$ & $\mathrm{N} 9-\mathrm{N} 10-\mathrm{C} 12-\mathrm{C} 10$ & $176.7(3)$ \\
\hline $\mathrm{C} 12-\mathrm{N} 7-\mathrm{N} 8-\mathrm{C} 13$ & $178.5(3)$ & $\mathrm{C} 9-\mathrm{C} 10-\mathrm{C} 12-\mathrm{N} 7$ & $140.6(3)$ \\
\hline $\mathrm{N} 7-\mathrm{N} 8-\mathrm{N} 9-\mathrm{N} 10$ & $-0.1(3)$ & $\mathrm{C} 8-\mathrm{C} 10-\mathrm{C} 12-\mathrm{N} 7$ & $-39.5(5)$ \\
\hline $\mathrm{C} 13-\mathrm{N} 8-\mathrm{N} 9-\mathrm{N} 10$ & $-178.4(3)$ & $\mathrm{C} 9-\mathrm{C} 10-\mathrm{C} 12-\mathrm{N} 10$ & $-35.4(4)$ \\
\hline $\mathrm{N} 8-\mathrm{N} 9-\mathrm{N} 10-\mathrm{C} 12$ & $-0.1(3)$ & $\mathrm{C} 8-\mathrm{C} 10-\mathrm{C} 12-\mathrm{N} 10$ & $144.6(3)$ \\
\hline $\mathrm{O} 10-\mathrm{S} 2-\mathrm{N} 14-\mathrm{C} 20$ & $-62.4(2)$ & $\mathrm{C} 14-\mathrm{O} 6-\mathrm{C} 15-\mathrm{N} 11$ & $174.7(2)$ \\
\hline $\mathrm{O} 9-\mathrm{S} 2-\mathrm{N} 14-\mathrm{C} 20$ & $167.96(19)$ & $\mathrm{C} 14-\mathrm{O} 6-\mathrm{C} 15-\mathrm{C} 16$ & $-5.5(3)$ \\
\hline $\mathrm{C} 21-\mathrm{S} 2-\mathrm{N} 14-\mathrm{C} 20$ & $53.4(2)$ & $\mathrm{C} 18-\mathrm{N} 11-\mathrm{C} 15-\mathrm{O} 6$ & $-179.3(2)$ \\
\hline $\mathrm{C} 21-\mathrm{N} 15-\mathrm{N} 16-\mathrm{C} 22$ & $0.2(3)$ & $\mathrm{C} 18-\mathrm{N} 11-\mathrm{C} 15-\mathrm{C} 16$ & $0.9(3)$ \\
\hline $\mathrm{C} 24-\mathrm{N} 15-\mathrm{N} 16-\mathrm{C} 22$ & $178.3(2)$ & $\mathrm{O} 6-\mathrm{C} 15-\mathrm{C} 16-\mathrm{C} 17$ & $-177.9(2)$ \\
\hline $\mathrm{C} 25-\mathrm{N} 17-\mathrm{N} 18-\mathrm{N} 19$ & $0.3(2)$ & $\mathrm{N} 11-\mathrm{C} 15-\mathrm{C} 16-\mathrm{C} 17$ & $1.9(4)$ \\
\hline $\mathrm{C} 25-\mathrm{N} 17-\mathrm{N} 18-\mathrm{C} 26$ & $177.8(2)$ & $\mathrm{C} 18-\mathrm{N} 12-\mathrm{C} 17-\mathrm{O} 7$ & $-179.8(2)$ \\
\hline $\mathrm{N} 17-\mathrm{N} 18-\mathrm{N} 19-\mathrm{N} 20$ & $-0.2(3)$ & $\mathrm{C} 18-\mathrm{N} 12-\mathrm{C} 17-\mathrm{C} 16$ & $1.6(3)$ \\
\hline $\mathrm{C} 26-\mathrm{N} 18-\mathrm{N} 19-\mathrm{N} 20$ & $-177.7(2)$ & $\mathrm{C} 19-\mathrm{O} 7-\mathrm{C} 17-\mathrm{N} 12$ & $3.8(3)$ \\
\hline $\mathrm{N} 18-\mathrm{N} 19-\mathrm{N} 20-\mathrm{C} 25$ & $0.0(2)$ & $\mathrm{C} 19-\mathrm{O} 7-\mathrm{C} 17-\mathrm{C} 16$ & $-177.5(2)$ \\
\hline $\mathrm{C} 1-\mathrm{O} 1-\mathrm{C} 2-\mathrm{N} 1$ & $7.6(3)$ & $\mathrm{C} 15-\mathrm{C} 16-\mathrm{C} 17-\mathrm{N} 12$ & $-3.2(4)$ \\
\hline $\mathrm{C} 1-\mathrm{O} 1-\mathrm{C} 2-\mathrm{C} 3$ & $-174.1(2)$ & $\mathrm{C} 15-\mathrm{C} 16-\mathrm{C} 17-\mathrm{O} 7$ & $178.2(2)$ \\
\hline $\mathrm{C} 5-\mathrm{N} 1-\mathrm{C} 2-\mathrm{O} 1$ & $175.3(2)$ & $\mathrm{C} 15-\mathrm{N} 11-\mathrm{C} 18-\mathrm{N} 12$ & $-2.9(4)$ \\
\hline $\mathrm{C} 5-\mathrm{N} 1-\mathrm{C} 2-\mathrm{C} 3$ & $-2.9(3)$ & $\mathrm{C} 15-\mathrm{N} 11-\mathrm{C} 18-\mathrm{N} 13$ & $176.3(2)$ \\
\hline $\mathrm{O} 1-\mathrm{C} 2-\mathrm{C} 3-\mathrm{C} 4$ & $-174.8(2)$ & $\mathrm{C} 17-\mathrm{N} 12-\mathrm{C} 18-\mathrm{N} 11$ & $1.7(4)$ \\
\hline $\mathrm{N} 1-\mathrm{C} 2-\mathrm{C} 3-\mathrm{C} 4$ & $3.4(4)$ & $\mathrm{C} 17-\mathrm{N} 12-\mathrm{C} 18-\mathrm{N} 13$ & $-177.5(2)$ \\
\hline $\mathrm{C} 6-\mathrm{O} 2-\mathrm{C} 4-\mathrm{N} 2$ & $-1.9(3)$ & $\mathrm{C} 20-\mathrm{N} 13-\mathrm{C} 18-\mathrm{N} 11$ & $176.5(2)$ \\
\hline $\mathrm{C} 6-\mathrm{O} 2-\mathrm{C} 4-\mathrm{C} 3$ & $178.0(2)$ & $\mathrm{C} 20-\mathrm{N} 13-\mathrm{C} 18-\mathrm{N} 12$ & $-4.2(4)$ \\
\hline $\mathrm{C} 5-\mathrm{N} 2-\mathrm{C} 4-\mathrm{O} 2$ & $179.3(2)$ & $\mathrm{S} 2-\mathrm{N} 14-\mathrm{C} 20-\mathrm{O} 8$ & $7.5(3)$ \\
\hline $\mathrm{C} 5-\mathrm{N} 2-\mathrm{C} 4-\mathrm{C} 3$ & $-0.6(3)$ & $\mathrm{S} 2-\mathrm{N} 14-\mathrm{C} 20-\mathrm{N} 13$ & $-171.02(17)$ \\
\hline $\mathrm{C} 2-\mathrm{C} 3-\mathrm{C} 4-\mathrm{O} 2$ & $178.6(2)$ & $\mathrm{C} 18-\mathrm{N} 13-\mathrm{C} 20-\mathrm{O} 8$ & $179.7(2)$ \\
\hline $\mathrm{C} 2-\mathrm{C} 3-\mathrm{C} 4-\mathrm{N} 2$ & $-1.5(4)$ & $\mathrm{C} 18-\mathrm{N} 13-\mathrm{C} 20-\mathrm{N} 14$ & $-1.8(4)$ \\
\hline $\mathrm{C} 2-\mathrm{N} 1-\mathrm{C} 5-\mathrm{N} 2$ & $0.5(4)$ & $\mathrm{N} 16-\mathrm{N} 15-\mathrm{C} 21-\mathrm{C} 23$ & $-0.4(3)$ \\
\hline $\mathrm{C} 2-\mathrm{N} 1-\mathrm{C} 5-\mathrm{N} 3$ & $-178.47(19)$ & $\mathrm{C} 24-\mathrm{N} 15-\mathrm{C} 21-\mathrm{C} 23$ & $-178.2(2)$ \\
\hline $\mathrm{C} 4-\mathrm{N} 2-\mathrm{C} 5-\mathrm{N} 1$ & $1.2(4)$ & N16-N15-C21-S2 & $-179.42(17)$ \\
\hline $\mathrm{C} 4-\mathrm{N} 2-\mathrm{C} 5-\mathrm{N} 3$ & $-179.9(2)$ & $\mathrm{C} 24-\mathrm{N} 15-\mathrm{C} 21-\mathrm{S} 2$ & $2.8(4)$ \\
\hline $\mathrm{C} 7-\mathrm{N} 3-\mathrm{C} 5-\mathrm{N} 1$ & $171.6(2)$ & $\mathrm{O} 10-\mathrm{S} 2-\mathrm{C} 21-\mathrm{N} 15$ & $13.3(2)$ \\
\hline $\mathrm{C} 7-\mathrm{N} 3-\mathrm{C} 5-\mathrm{N} 2$ & $-7.5(4)$ & $\mathrm{O} 9-\mathrm{S} 2-\mathrm{C} 21-\mathrm{N} 15$ & $145.26(19)$ \\
\hline $\mathrm{S} 1-\mathrm{N} 4-\mathrm{C} 7-\mathrm{O} 3$ & $-0.4(4)$ & $\mathrm{N} 14-\mathrm{S} 2-\mathrm{C} 21-\mathrm{N} 15$ & $-104.2(2)$ \\
\hline $\mathrm{S} 1-\mathrm{N} 4-\mathrm{C} 7-\mathrm{N} 3$ & $-178.80(17)$ & $\mathrm{O} 10-\mathrm{S} 2-\mathrm{C} 21-\mathrm{C} 23$ & $-165.5(2)$ \\
\hline $\mathrm{C} 5-\mathrm{N} 3-\mathrm{C} 7-\mathrm{O} 3$ & $-177.1(2)$ & $\mathrm{O} 9-\mathrm{S} 2-\mathrm{C} 21-\mathrm{C} 23$ & $-33.5(3)$ \\
\hline $\mathrm{C} 5-\mathrm{N} 3-\mathrm{C} 7-\mathrm{N} 4$ & $1.4(4)$ & $\mathrm{N} 14-\mathrm{S} 2-\mathrm{C} 21-\mathrm{C} 23$ & $77.0(2)$ \\
\hline $\mathrm{N} 6-\mathrm{N} 5-\mathrm{C} 8-\mathrm{C} 10$ & $1.2(3)$ & $\mathrm{N} 15-\mathrm{N} 16-\mathrm{C} 22-\mathrm{C} 23$ & $0.1(3)$ \\
\hline $\mathrm{C} 11-\mathrm{N} 5-\mathrm{C} 8-\mathrm{C} 10$ & $179.8(3)$ & $\mathrm{N} 15-\mathrm{C} 21-\mathrm{C} 23-\mathrm{C} 22$ & $0.4(3)$ \\
\hline $\mathrm{N} 6-\mathrm{N} 5-\mathrm{C} 8-\mathrm{S} 1$ & $-174.8(2)$ & $\mathrm{S} 2-\mathrm{C} 21-\mathrm{C} 23-\mathrm{C} 22$ & $179.38(19)$ \\
\hline $\mathrm{C} 11-\mathrm{N} 5-\mathrm{C} 8-\mathrm{S} 1$ & $3.8(4)$ & $\mathrm{N} 15-\mathrm{C} 21-\mathrm{C} 23-\mathrm{C} 25$ & $-175.6(2)$ \\
\hline
\end{tabular}




$\begin{array}{llll}\mathrm{O} 5-\mathrm{S} 1-\mathrm{C} 8-\mathrm{N} 5 & 16.9(3) & \mathrm{S} 2-\mathrm{C} 21-\mathrm{C} 23-\mathrm{C} 25 & 3.4(4) \\ \mathrm{O} 4-\mathrm{S} 1-\mathrm{C} 8-\mathrm{N} 5 & 148.5(2) & \mathrm{N} 16-\mathrm{C} 22-\mathrm{C} 23-\mathrm{C} 21 & -0.3(3) \\ \mathrm{N} 4-\mathrm{S} 1-\mathrm{C} 8-\mathrm{N} 5 & -101.5(2) & \mathrm{N} 16-\mathrm{C} 22-\mathrm{C} 23-\mathrm{C} 25 & 176.1(2) \\ \mathrm{O} 5-\mathrm{S} 1-\mathrm{C} 8-\mathrm{C} 10 & -158.3(2) & \mathrm{N} 18-\mathrm{N} 17-\mathrm{C} 25-\mathrm{N} 20 & -0.2(3) \\ \mathrm{O} 4-\mathrm{S} 1-\mathrm{C} 8-\mathrm{C} 10 & -26.6(3) & \mathrm{N} 18-\mathrm{N} 17-\mathrm{C} 25-\mathrm{C} 23 & 179.0(2) \\ \mathrm{N} 4-\mathrm{S} 1-\mathrm{C} 8-\mathrm{C} 10 & 83.4(3) & \mathrm{N} 19-\mathrm{N} 20-\mathrm{C} 25-\mathrm{N} 17 & 0.1(3) \\ \mathrm{N} 5-\mathrm{N} 6-\mathrm{C} 9-\mathrm{C} 10 & 0.1(3) & \mathrm{N} 19-\mathrm{N} 20-\mathrm{C} 25-\mathrm{C} 23 & -179.0(2) \\ \mathrm{N} 6-\mathrm{C} 9-\mathrm{C} 10-\mathrm{C} 8 & 0.5(3) & \mathrm{C} 21-\mathrm{C} 23-\mathrm{C} 25-\mathrm{N} 17 & 151.6(3) \\ \mathrm{N} 6-\mathrm{C} 9-\mathrm{C} 10-\mathrm{C} 12 & -179.5(3) & \mathrm{C} 22-\mathrm{C} 23-\mathrm{C} 25-\mathrm{N} 17 & -23.7(4) \\ \mathrm{N} 5-\mathrm{C} 8-\mathrm{C} 10-\mathrm{C} 9 & -1.0(3) & \mathrm{C} 21-\mathrm{C} 23-\mathrm{C} 25-\mathrm{N} 20 & -29.3(4) \\ \mathrm{S} 1-\mathrm{C} 8-\mathrm{C} 10-\mathrm{C} 9 & 174.8(2) & \mathrm{C} 22-\mathrm{C} 23-\mathrm{C} 25-\mathrm{N} 20 & 155.4(2) \\ \end{array}$

Hydrogen-bond geometry $\left(A,{ }^{\circ}\right)$

\begin{tabular}{lllll}
\hline$D-\mathrm{H} \cdots A$ & $D-\mathrm{H}$ & $\mathrm{H} \cdots A$ & $D \cdots A$ & $D-\mathrm{H}^{\cdots} A$ \\
\hline $\mathrm{N} 3-\mathrm{H} 3 N \cdots \mathrm{N} 11^{\mathrm{i}}$ & 0.88 & 2.65 & $3.494(3)$ & 162 \\
$\mathrm{~N} 4-\mathrm{H} 4 N \cdots \mathrm{N} 2$ & 0.88 & 1.89 & $2.611(3)$ & 138 \\
$\mathrm{~N} 13-\mathrm{H} 13 N \cdots \mathrm{O} 3^{\mathrm{i}}$ & 0.88 & 2.04 & $2.844(2)$ & 151 \\
$\mathrm{~N} 14-\mathrm{H} 14 N \cdots \mathrm{N} 12$ & 0.88 & 1.95 & $2.636(3)$ & 134 \\
$\mathrm{C} 1-\mathrm{H} 1 C \cdots \mathrm{O} 8^{\mathrm{ii}}$ & 0.98 & 2.58 & $3.559(3)$ & 178 \\
$\mathrm{C} 11-\mathrm{H} 11 B \cdots \mathrm{O} 3$ & 0.98 & 2.38 & $3.236(3)$ & 146 \\
$\mathrm{C} 11-\mathrm{H} 11 B \cdots \mathrm{O} 8^{\mathrm{i}}$ & 0.98 & 2.57 & $3.278(3)$ & 129 \\
$\mathrm{C} 11-\mathrm{H} 11 C \cdots \mathrm{N} 10^{\mathrm{iii}}$ & 0.98 & 2.58 & $3.290(4)$ & 130 \\
$\mathrm{C} 13-\mathrm{H} 13 A \cdots \mathrm{O} 9^{\mathrm{iv}}$ & 0.98 & 2.40 & $3.374(4)$ & 175 \\
$\mathrm{C} 14-\mathrm{H} 14 A \cdots \mathrm{O} 5^{\mathrm{v}}$ & 0.98 & 2.39 & $3.335(3)$ & 162 \\
$\mathrm{C} 22-\mathrm{H} 22 \cdots \mathrm{N} 17^{\mathrm{vi}}$ & 0.95 & 2.57 & $3.435(3)$ & 152 \\
$\mathrm{C} 24-\mathrm{H} 24 B \cdots \mathrm{O} 8$ & 0.98 & 2.33 & $3.080(3)$ & 133 \\
$\mathrm{C} 26-\mathrm{H} 26 B \cdots \mathrm{N} 16^{\mathrm{vi}}$ & 0.98 & 2.52 & $3.376(3)$ & 146 \\
& & & &
\end{tabular}

Symmetry codes: (i) $-x+1,-y+1,-z+1$; (ii) $x-1, y, z$; (iii) $-x+1,-y+1,-z$; (iv) $-x+2,-y,-z+1$; (v) $x+1, y, z$; (vi) $-x+1,-y,-z+2$. 